\section{Role of distress in delusion formation*}

\author{
MANON HANSSEN, LYDIA KRABBENDAM, RON DE GRAAF, \\ WILMA VOLLEBERGH and JIM VAN OS
}

\section{Background Contemporary cognitive psychological theories suggest that distress plays a mediating role in delusion formation.}

\begin{abstract}
Aims To study the amplifying role of distress from early perceptual intrusions to delusion formation.
\end{abstract}

Method A general population sample of 7076 individuals was interviewed with the Composite International Diagnostic Interview (CIDI) in 1996 (baseline), 1997 (TI) and 1999 (T2). At T2, clinicians also scored the Brief Psychiatric Rating Scale (BPRS) item 'unusual thought content'. Analyses compared hallucinatory experiences with and without subjective distress at baseline for risk of delusion formation at follow-up.

Results Individuals experiencing hallucinations with distress, compared with those without distress had a fourfold increased risk of subsequent delusion formation.

Conclusions This finding corroborates the hypothesis that distress associated with early perceptual intrusions serves as a catalyst in the development of delusions.

Declaration of interest None. Funding detailed in Acknowledgements.

* Paper presented at theThird International Early Psychosis Conference, Copenhagen, Denmark, September 2002.
Hallucinations and delusions tend to cooccur both in clinical and in non-clinical samples (Liddle \& Barnes, 1990; Johnstone \& Frith, 1996; van Os et al, 2000). One possible explanation for this association may be that the experience of hallucinations gives rise to secondary delusional interpretations (De Clérambault, 1942; Maher, 1988). Little is known, however, about the factors that mediate the transition from hallucinatory experience to delusional interpretation. Recent psychological hypotheses on delusion formation emphasise the role of attributional style, distress and worry in the aetiology and maintenance of delusions (Garety et al, 2001). Thus, the experience of voices or visions may lead to full-blown delusional ideation, when it is attributed to an external source or when it is given personal significance.

In an earlier study, for example, it was reported that delusion formation in adolescents hearing voices was mediated by, among other factors, attributions of externality, perceived power over the person and emotional appraisals (Escher et al, 2002). We wished to extend these results to a large general population sample that had no previous evidence of delusional ideation. It was hypothesised that people experiencing hallucinations with distress at baseline, compared with those without, would show a greater risk of developing delusions over the follow-up period.

\section{METHOD}

\section{Procedure, instruments and sample}

The Netherlands Mental Health Survey and Incidence Study (NEMESIS study) is a large general population study with three measurement points (hereafter: baseline, T1 and T2) in 1996, 1997 and 1999. At the three measurement points, respectively 7076, 5618 and 4848 individuals aged
18-64 years participated. Trained lay interviewers in all three measurements applied the Composite International Diagnostic Interview (CIDI; Smeets \& Dingemans, 1993). The CIDI has 17 psychosis items (13 delusions, 4 hallucinations) with six possible ratings for each psychosis item: (1) no psychotic experience; (2) no clinically relevant psychotic experience; the individual is not experiencing distress and is not seeking help; (3) and (4) psychotic experience is the result of drug misuse or somatic disease; (5) true psychiatric symptom; individual experiences distress and/or seeks help; (6) what appears to be a true psychotic symptom may not be a real symptom because there appears to be some plausible explanation for it, i.e. it may actually exist.

At baseline, the lay interviewers enquired about lifetime presence of symptoms and at the two follow-up measurements the period between the measurements was assessed. For the current analysis, ratings of 2 and 5 on the four hallucination items at baseline, both indicative of the presence of a psychotic experience in the absence of doubt or secondary cause but crucially different in terms of subjective distress and help-seeking behaviour, were included in the analyses. The distinction between the ratings of 2 and 5 was validated in a previous study (van Os et al, 2001). The four hallucination items encompassed all possible hallucinatory modalities.

At baseline and T2, attempts were made to clinically validate the lay interviewer-administered CIDI interview. Each time, when at baseline (possible) psychotic symptoms (CIDI rating of 5 or 6 ) were detected in the NEMESIS study, a psychiatric clinician conducted clinical re-interviews over the telephone by using the Structured Clinical Interview for DSM-III-R (SCID; Spitzer et al, 1992). If a clinician did not agree with the psychosis rating of the trained lay interviewer, the psychosis rating was changed to the rating of the clinician. All DSM-III-R diagnoses in the NEMESIS study are based on these corrected ratings. At baseline, $47.2 \%$ of the eligible individuals were actually interviewed. The probability of a selection bias was assessed in a previous study and deemed unlikely (Hanssen et al, 2003). At T2, all individuals with a rating of 2, 5 or 6 on any CIDI psychosis item were administered a clinical re-interview over the telephone by an experienced clinician. The proportion of eligible individuals who were successfully 
re-interviewed was $74.4 \%$. At $\mathrm{T} 2$, the clinician also scored the 'unusual though content' item of the Brief Psychiatric Rating Scale (BPRS; Lukoff et al, 1986). The BPRS symptom items are rated on a seven-point scale on the basis of frequency of the symptom and functional impairment. Ratings 2-3 represent a non-pathological form of the symptoms and ratings 4-7 represent a pathological form (Lukoff et al, 1986). For a more detailed description of the NEMESIS study see Bijl et al (1998) and van Os et al (2001).

\section{Analyses: the development of delusions}

The study sample at $\mathrm{T} 2$ was restricted to individuals who did not report delusions at baseline and again at T1 (i.e. had no ratings of 2 or 5 on any of the CIDI delusion items at baseline and T1) in order to skew the sample towards people with true incident delusions at T2 (original sample at T2: $n=4848$; sample restricted to those without delusions at baseline and T1, and non-missing data on the delusion variable at T2, hereafter referred to as 'risk set': $n=4236$; risk set and non-missing covariates: $n=4181$ ).

All analyses were carried out with Stata version 7 Special Edition (StataCorp, 2001). Logistic regression analysis was performed with the BPRS 'unusual thought content' item measured at T2 as a dependent variable (score 1 rated absent and score $>1$ rated present) and the baseline CIDI ratings on hallucinations with and without distress (entered as two dummy variables) as independent variables. To account for possible confounding variables, we controlled for the following a priori chosen covariates, guided by previous findings in this sample (van Os et al, 2000, 2001, 2002; Krabbendam et al, 2002; Janssen et al, 2003): age (5 categories), gender, urbanicity (3 levels), ethnic group (0: person and both parents born in the Netherlands and 1: other), neuroticism (Ormel, 1980), experience of discrimination (a 6-item questionnaire measuring experience of discrimination regarding the colour of skin or ethnicity, gender, age, appearance, handicap and sexual orientation), experience of abuse before the age of 16 years (a 4-item questionnaire), educational level (4 levels), unemployment and single marital status. The effect sizes of baseline hallucinations with and without distress on risk for delusion formation at $\mathrm{T} 2$ were compared using the Wald test. In order to exclude misclassification at the lower end of the BPRS scoring range of the unusual thought content item, analyses were repeated with a more stringent definition of the BPRS 'unusual thought content' item as a dependent variable, i.e. a score $>2$. Finally, in an attempt to clinically validate any findings, analyses were repeated using the clinical definition of delusions, namely a score $>3$ on the BPRS item 'unusual thought content', which is indicative of the level of pathology in terms of functional impairment (Lukoff et al, 1986).

\section{Sensitivity analyses}

Sensitivity analyses were conducted to examine whether differential attrition in the sample as a whole (7076 at baseline, 4848 at T2) could have biased the findings. This was done by multiple imputation of missing values of delusional ideation at $\mathrm{T} 2$ ( $n=1962$ missing, $31.4 \%$ ) using the HOTDECK command in Stata. The HOTDECK procedure is used several times within a multiple imputation sequence as missing data are imputed stochastically rather than deterministically. A total of 1000 imputation sequences were run, yielding 1000 data-sets in which the regression coefficients were estimated within the HOTDECK procedure. Imputation of missing values was stratified by known risk factors of psychosis (van Os et al, 2000, 2001, 2002; Krabbendam et al, 2002; Janssen et al, 2003), namely age, gender, urbanicity, ethnic group, neuroticism, experience of discrimination, experience of abuse before the age of 16 years, educational level, unemployment and single marital status. The HOTDECK procedure replaces missing values in the relevant variables by values randomly sampled from complete lines in the same stratum. Individuals who had delusions at baseline and at T1 were again excluded from these analyses $(n=831)$.

\section{RESULTS}

\section{Data}

At baseline and limited to the risk set $(n=4236), 161$ individuals (males: $34.8 \%$ ) reported lifetime occurrence of hallucinations without distress, whereas 32 (males: $21.9 \%$ ) reported hallucinations with distress. Five subjects reported both types of hallucinations.

In the risk set $(n=4236)$, visual, auditory, olfactory and tactile hallucinations without distress were reported by 79 (1.9\%), $26(0.6 \%), 34(0.8 \%)$ and 60 $(1.4 \%)$ individuals, respectively. Visual, auditory, olfactory and tactile hallucinations accompanied by distress were present in $19(0.5 \%), 8(0.2 \%), 5(0.1 \%)$ and 8 $(0.2 \%)$, respectively. In the group of 161 individuals who reported hallucinatory experiences without distress, the proportions of visual, auditory, olfactory and tactile hallucinations were $49.1 \% \quad(79 /$ $161), 16.2 \%$ (26/161), $21.1 \%$ (34/161) and $37.3 \%(60 / 161)$, respectively. In the distress group $(n=32)$ these proportions were $59.4 \% \quad(19 / 32), \quad 25.0 \% \quad(8 / 32), \quad 15.6 \%$ (5/32) and $25.0 \%$ (8/32), respectively.

Thirty-seven individuals in the risk set (males: 35\%) had developed delusions (BPRS $>1$ ) at T2, 16 of these 37 (males: $56 \%$ ) had developed delusions with a BPRS score $>2$, and 7 (males: $71 \%$ ) of these 16 had developed clinical delusions according to the BPRS definition (BPRS $>3$ ).

\section{Distress and delusion formation}

The risk for delusion formation at T2 was five times greater in the individuals who at baseline were distressed by their hallucinations (6 out of 32 persons reporting hallucinations with distress developed delusions (BPRS $>1$ : OR $=25.0,95 \%$ CI 9.3$67.8)$ than in the individuals who reported hallucinations without distress (7 out of 161 persons reporting hallucinations without distress developed delusions (BPRS $>1$ : $\mathrm{OR}=4.9,95 \%$ CI 2.0-11.9) (Table 1). This difference was statistically significant $\left(\chi^{2}=5.2\right.$, d.f. $\left.=1, P=0.02\right)$. After adjustment for the covariates, the difference remained robust $\left(\chi^{2}=3.8\right.$, d.f. $=1, P=0.05$ ) (Table 1 ).

Results were similar using the more stringent definition of delusions (BPRS $>2$ ), with again a highly significant difference in effect size $\left(\chi^{2}=8.7, \quad\right.$ d.f. $\left.=1, \quad P=0.003\right)$ (Table 1), also after adjustment of covariates. Similar results were apparent for the clinical definition of delusions: no individuals with hallucinations without distress developed clinically relevant delusional ideation, whereas the risk was very high in those whose hallucinatory experiences were accompanied by distress (Table 1).

\section{Sensitivity analyses}

Using 1000 imputation sequences in which missing values of the outcome of delusions at T2 were imputed stochastically, the estimated effect size for baseline hallucinations with distress was $(\mathrm{OR}=18.3,95 \%$ 
Table I Comparison between hallucinations with and without distress regarding the formation of delusions 3 years later

\begin{tabular}{lllll}
\hline Covariates & \multicolumn{1}{c}{ Baseline } & \multicolumn{1}{c}{ Baseline } & $\chi^{2}$ & d.f.
\end{tabular}

\begin{tabular}{|c|c|c|c|c|c|}
\hline \multicolumn{6}{|l|}{ Unadjusted } \\
\hline T2: BPRS & $25.0(9.3-67.8)$ & $4.9(2.0-11.9)$ & 5.2 & I & 0.02 \\
\hline delusion $>$ I & $6 / 32$ & $7 / 161$ & & & \\
\hline T2: BPRS & $50.4(14.9-169.9)$ & $0.9(0.1-7.8)$ & 8.7 & I & 0.003 \\
\hline delusion $>2$ & $4 / 32$ & $1 / 161$ & & & \\
\hline T2: BPRS & $126.4(26.8-595.3)$ & $-^{2}$ & - & - & - \\
\hline delusion $>3$ & $3 / 32$ & $0 / 161$ & & & \\
\hline \multicolumn{6}{|l|}{ Adjusted } \\
\hline $\begin{array}{l}\text { T2: BPRS } \\
\text { delusion }>\text { I }\end{array}$ & I3.7 (4.4-42.4) & $3.2(1.2-8.4)$ & 3.8 & I & 0.05 \\
\hline +covariates & & & & & \\
\hline $\begin{array}{l}\text { T2: } \text { BPRS } \\
\text { delusion }>2 \\
\text { +covariates }\end{array}$ & $25.1(4.8-131.3)$ & $0.5(0.04-4.9)$ & 8.5 & I & 0.004 \\
\hline $\begin{array}{l}\text { T2: BPRS } \\
\text { delusion }>3 \\
\text { +covariates }\end{array}$ & $136.2(|3.1-| 4 \mid 4.3)$ & $-^{2}$ & - & - & - \\
\hline
\end{tabular}

BPRS, Brief Psychiatric Rating Scale.

I. Frequency means the number of individuals with delusion formation given the number of individuals with lifetime hallucinations with or without distress.

2. Predicts failure perfectly.

CI 5.6-60.2) and for baseline hallucinations without distress $(\mathrm{OR}=5.4,95 \% \mathrm{CI}$ 2.1-14.3), indicating a similar pattern of results.

\section{DISCUSSION}

The results show that those who experience negative emotional states associated with anomalous perceptual intrusions have a much greater risk of developing delusional ideation, including experiences of clinical relevance, than individuals who report similar experiences without distress.

Individuals reporting distress associated with their hallucinations did show a much greater risk for developing clinical delusions than those reporting hallucinations without distress. Our findings support the amplifying role of distress in current cognitive models of delusion formation (Freeman \& Garety, 1999; Birchwood et al, 2000; Morrison \& Baker, 2000; Garety et al, 2001). According to these models, feelings of uncontrollability and hopelessness associated with negative emotional states may contribute to the onset of delusional interpretations. Emotions may also make hallucinatory experiences personally significant or more intrusive, which in turn may trigger the individual to search for explanations of the experiences. The distress caused by hallucinatory experiences may in turn be related to the interpretation of the experience (Morrison \& Baker, 2000). The mechanism of delusion formation may depend on the initial interpretation individuals give to their unusual perceptual intrusions. If this initial interpretation leads to distress, the individual may be more prone to selective attentional processes and safety behaviours, diminishing the opportunity to test the accuracy of the psychotic experience (Garety et al, 2001), resulting in increased levels of delusional ideation.

The role of distress associated with unusual experiences may also be crucial to understand further transitions over the psychosis continuum. Peters et al (1999) measured delusional ideation in the general population as well as in those with delusions using the Peters et al Delusions Inventory (PDI). The PDI scores of the general population and the patients with delusions showed a large degree of overlap and nearly $10 \%$ of the general population scored above the mean of the group with delusions. However, compared with patients, the general population displayed significantly less distress, preoccupation and conviction regarding their unusual perceptual experiences and ideas.

The present study is limited to psychological factors, but biological factors may also be relevant in this regard. Hemsley's cognitive model hypothesises that the problem in schizophrenia encompasses the inability to integrate the moment-bymoment sensory input with stored memory (Hemsley, 1993, 1994). A neuronal circuit, including the limbic system, is proposed to be involved in this integration process. Distress can lead to an increased dopamine release that in turn may influence the functioning of these brain structures (Robinson \& Becker, 1986). Thus, a stress-induced dopaminergic response in humans could result in a heightened risk for positive psychotic symptoms in vulnerable persons, with possible subsequent sensitisation of dopaminergic response and persistence of delusional ideation (Laruelle, 2000).

In summary, the present findings have implications for early intervention in psychosis or psychosis-like experiences, and underline the significance of cognitivebehavioural therapy in treating psychotic disorders (Kingdon \& Turkington, 1994; Turkington \& Kingdon, 2000). If distress associated with hallucinations is involved in the development of delusions, ameliorating the distress may prevent the formation of delusions in some individuals. Cognitive-behavioural, anxiety-reducing and reappraisal techniques could be instrumental in preventing the development of delusions in those with anomalous experiences (McGorry et al, 2002; Morrison et al, 2002).

However, this work should be interpreted in the light of several potential limitations. First, distress was operationalised as feeling disturbed by the hallucinations and/or displaying help-seeking behaviour. Thus, the mediating role of distress in the development of delusions is a general one, as we did not have any information linking distress to content of or beliefs about hallucinations and/or delusions. Second, differential attrition in this longitudinal design could have biased the results. However, sensitivity analyses generated essentially similar results. Third, the present study monitored only one of the many mediating and maintaining factors proposed by Garety et al (2001). However, it was not possible to examine the role of many other important variables in the formation of delusions (e.g. externalising attributional biases, problems with selfmonitoring, dysfunctional schemas etc.), as we did not gather this information. Finally, the outcome 'unusual thought 
content' was very rare, affecting the precision with which we could estimate effect sizes.

\section{ACKNOWLEDGEMENTS}

This study was funded by the Dutch Department of Health.

\section{REFERENCES}

Bijl, R.V., van Zessen, G., Ravelli, A., et al (1998) The Netherlands Mental Health Survey and Incidence Study (NEMESIS): objectives and design. Social Psychiatry and Psychiatric Epidemiology, 33, 58I-586.

Birchwood, M., Meaden, A., Trower, P., et al (2000) The power and omnipotence of voices: subordination and entrapment by voices and significant others. Psychological Medicine, 30, 337-344.

De Clérambault, G. G. (1942) Oeuvre Psychiatrique (Vol. 2). Paris: Presses Universitaires de France.

Escher, S., Romme, M., Buiks, A., et al (2002) Formation of delusional ideation in adolescents hearing voices: A prospective study. American Journal of Medica Genetics, II4, 913-920.

Freeman, D. \& Garety, P. A. (1999) Worry, worry processes and dimensions of delusions: an exploratory investigation of a role for anxiety processes in the maintenance of delusional distress. Behavioural and Cognitive Psychotherapy, 27, 47-62.

Garety, P. A., Kuipers, E., Fowler, D., et al (200I) A cognitive model of the positive symptoms of psychosis. Psychological Medicine, 31, 189-195.

Hanssen, M., Bijl, R. V., Vollebergh, W., et al (2003) Self-reported psychotic experiences in the general population: a valid screening tool for DSM-III-R psychotic disorders? Acta Psychiatrica Scandinavica, 107, 369-377.

Hemsley, D. R. (1993) A simple (or simplistic?) cognitive model for schizophrenia. Behaviour Research and Therapy, 31, 633-645.

Hemsley, D. R. (1994) A cognitive model for schizophrenia and its possible neural basis. Acta Psychiatrica Scandinavica Supplementum, 384, 80-86.

Janssen, I., Hanssen, M., Bak, M., et al (2003)

Discrimination and delusional ideation. British journal of Psychiatry, 182, 7|-76.

Johnstone, E. C. \& Frith, C. D. (1996) Validation of three dimensions of schizophrenic symptoms in a large unselected sample of patients. Psychological Medicine, 26, 669-679.

Kingdon, D. \& Turkington, D. (1994) CognitiveBehavioral Therapy of Schizophrenia. Hove, UK: Lawrence Erlbaum.

Krabbendam, L., Janssen, I., Bak, M., et al (2002) Neuroticism and low self-esteem as risk factors for psychosis. Social Psychiatry and Psychiatric Epidemiology, 37. $1-6$

Laurelle, M. (2000) The role of endogenous sensitization in the pathophysiology of schizophrenia: implications from recent brain imaging studies. Brain Research Brain Research Reviews, 31, 37I-384.

Liddle, P. F. \& Barnes, T. R. (1990) Syndromes of chronic schizophrenia. British Journal of Psychiatry, I57, 558-561.

Lukoff, D., Nuechterlein, K. H. \& Ventura, J. (1986) Manual for Expanded Brief Psychiatric Rating Scale. Schizophrenia Bulletin, 12, 594-602.

\section{CLINICAL IMPLICATIONS}

Psychotic experiences are common and transitions to clinical disorder are in part determined by emotional factors.

- Not only the presence of an unusual perceptual experience in itself but also the emotional appraisal by the subject is an important risk factor for subsequent delusion formation.

- Cognitive-behavioural therapy, in the context of early hallucinatory experiences, may be more efficient when specifically targeting the distress generated by the experience.

\section{LIMITATIONS}

- The distress measures used in the present study were limited to 'feeling disturbed or displaying help-seeking behaviour'.

- The outcome under study was very rare, affecting the precision with which we could estimate effect sizes.

- The present study examined only one of the proposed mediating and maintaining factors in the cognitive model of Garety et al (200I).

MANON HANSSEN, MA, PhD, LYDIA KRABBENDAM, MA, PhD, Department of Psychiatry and Neuropsychology, Maastricht University; RON de GRAAF, MA, PhD,WILMA VOLLEBERGH, MA, PhD, The Netherlands Institute of Mental Health and Addiction, Utrecht; JIM van OS, MD, PhD, MRCPsych, Department of Psychiatry and Neuropsychology, Maastricht University, The Netherlands and Division of Psychological Medicine, Institute of Psychiatry, London, UK

Correspondence: Professor Jim van Os, Department of Psychiatry and Neuropsychology, Maastricht University, PO Box 616 (DRT I0), 6200MD Maastricht, The Netherlands. Tel: +3I 43387 5443; fax: +31 43387 5444; e-mail: j.vanos@sp.unimaas.nl

Maher, B. A. (1988) Anomalous experience and delusional thinking: the logic of explanations. In Delusional Beliefs (eds T. F. Oltmanns \& B. Maher) pp. 15-33. New York: John Wiley.

McGorry, P. D., Yung, A. R., Phillips, L. J., et al (2002) Randomized controlled trial of interventions designed to reduce the risk of progression to first-episode psychosis in a clinical sample with subthreshold symptoms. Archives of General Psychiatry, 59, 921-928.

Morrison, A. P. \& Baker, C. A. (2000) Intrusive thoughts and auditory hallucinations: a comparative study of intrusions in psychosis. Behaviour Research and Therapy, 38, 1097-1106.

Morrison, A. P., Bentall, R. P., French, P., et al (2002) Randomised controlled trial of early detection and cognitive therapy for preventing transition to psychosis in high-risk individuals. Study design and interim analysis of transition rate and psychological risk factors. British Journal of Psychiatry 18I (suppl. 43), s78-s84.

Ormel, J. (1980) Moeite met leven of een moeilijk leven? Groningen: University of Groningen.

Peters, E. R., Joseph, S. A. \& Garety, P. A. (1999) Measurement of delusional ideation in the normal population: introducing the PDI (Peters et al. Delusions Inventory). Schizophrenia Bulletin, 25, 553-576.

Robinson, T. E. \& Becker, J. B. (1986) Enduring changes in brain and behavior produced by chronic amphetamine administration: a review and evaluation of animal models of amphetamine psychosis. Brain Research, 396, 157-198.

Smeets, R. M. W. \& Dingemans, P. M. A. J. (1993) Composite International Diagnostic Interview (CIDI), Version I.I (in Dutch). Amsterdam/Geneva: World Health Organization.

Spitzer, R. L., Williams, J. B., Gibbon, M., et al (1992) The Structured Clinical Interview for DSM-III-R (SCID): I. History, rationale, and description. Archives of General Psychiatry, 49, 624-629.

StataCorp (200I) Stata Statistical Software: Release 7.0. College Station, TX: Stata Corporation.

Turkington, D. \& Kingdon, D. (2000) Cognitivebehavioural techniques for general psychiatrists in the management of patients with psychoses. British Journal of Psychiatry, 177, 101-106.

van Os, J., Hanssen, M., Bijl, R.V., et al (2000) Strauss (1969) revisited: a psychosis continuum in the general population? Schizophrenia Research, 45, II-20.

van Os, J., Hanssen, M., Bijl, R.V., et al (200I) Prevalence of psychotic disorder and community level of psychotic symptoms: an urban-rural comparison. Archives of General Psychiatry, 58, 663-668.

van Os, J., Hanssen, M., De Graaf, R., et al (2002) Does the urban environment independently increase the risk for both negative and positive features of psychosis? Social Psychiatry and Psychiatric Epidemiology, 37, $460-464$. 